\title{
Cross-linked acrylic hydrogel for the controlled delivery of hydrophobic drugs in cancer therapy
}

This article was published in the following Dove Press journal:

International Journal of Nanomedicine

26 July 2012

Number of times this article has been viewed

\section{G Deepa'}

Arun Kumar T Thulasidasan ${ }^{2}$

Ruby John Anto ${ }^{2}$

J Jisha Pillai'

GS Vinod Kumar'

'Chemical Biology, ${ }^{2}$ Division of Cancer Research, Rajiv Gandhi Centre for Biotechnology, Thiruvananthapuram, Kerala, India
Correspondence: GSVinod Kumar Chemical Biology, Molecular Medicine Division, Rajiv Gandhi Centre for Biotechnology, Poojappura, Thiruvananthapuram-695 014, Kerala, India

$\mathrm{Tel}+9 \mid$ 47| 2529526

Fax +9| 47| 2348096

Email gsvinod@rgcb.res.in
Objective: To investigate cross-linked hydrogels prepared via inverse emulsion polymerization to entrap poorly aqueous soluble drugs. Polyethylene glycol cross-linked acrylic polymers were synthesized and the loading and release of curcumin, a model hydrophobic drug, was investigated.

Methods: Physicochemical characteristics of hydrogels were studied with ${ }^{13} \mathrm{C}$ nuclear magnetic resonance, Fourier transform infrared spectroscopy, transmission electron microscopy, scanning electron microscopy, differential scanning calorimetry, and swelling. Polymerization of the acrylic acid with cross-linked polyethylene glycol diacrylate was characterized with ${ }^{13} \mathrm{C}$ nuclear magnetic resonance imaging and Fourier transform infrared spectroscopy.

Results: The in vitro release rate of curcumin showed that there was a sustained release from the hydrogel with increased cross-linking; the release rate depended on the $\mathrm{pH}$ of the releasing medium. Intracellular and cytotoxicity studies were carried out in human cervical cancer cell lines.

Conclusion: The results suggest cross-linked acrylic polymers can be used as efficient vectors for $\mathrm{pH}$-sensitive, controlled delivery of hydrophobic drugs.

Keywords: curcumin, cross-linked polyethylene glycol, polyacrylic acid, nanogel, cross-linking combinations, HeLa

\section{Introduction}

Hydrogels are three-dimensional polymer networks that exhibit a semisolid morphology and can absorb a large amount of water. The water content of hydrogels occurs due to the presence of ionizable functional groups, which affects characteristics such as permeability, mechanical stability, and biocompatibility. ${ }^{1}$ Hydrogels' highly porous structure can easily be tuned by controlling the density of cross-links in the gel matrix and the affinity of the hydrogels for the aqueous environment in which they are swollen. Their porosity also permits loading of drugs into the gel matrix and subsequent drug release at a rate dependent on the diffusion coefficient of the small molecule or macromolecule through the gel network. Hydrogels are extremely useful for application in various fields such as drug delivery and tissue engineering due to biocompatibility, stimuli responsive behavior, and susceptibility for chemical modification. ${ }^{2-4}$ Biodegradable polymer systems for controlled-release drug delivery have been extensively studied because an invasive technique such as surgery is not required after their use..$^{5-7}$ Especially, biodegradable hydrogels have been represented as an attractive drug formulation because of their advantages such as biocompatibility, ${ }^{8}$ high responsibility for specific degradation, and a feasible approach for incorporating drugs in matrices. 
Hydrogel-based devices belong to the group of swelling-controlled drug delivery systems. ${ }^{9}$ When the polymer network comes in contact with aqueous solutions, the thermodynamic compatibility of the polymer chains and water causes the polymer to swell. ${ }^{10}$ As water penetrates inside the glassy network, the glass temperature of the polymer decreases, and the hydrogel becomes rubbery. In the case of nonswelling-controlled delivery systems, the relaxation rate of the polymer is very slow compared with the water transport inside the hydrogel. Hydrogels have been extensively exploited for biomedical applications due to their high water content, which imparts excellent biocompatibility. ${ }^{11}$ The high water content and large pore sizes of most hydrogels often result in relatively rapid drug release, over a few hours or days. Hydrogels have been reported as novel vectors for controlled drug-release systems since they exhibit dramatic changes in their swelling behavior, ${ }^{12}$ network structure, permeability, or mechanical strength in response to different stimuli. Hydrogels can be prepared from natural and synthetic polymers. Those prepared from natural polymers possess inherent bioactive properties whereas those prepared from synthetic polymers have well-defined structures that can be modified to yield biocompatibility and functionality that can be tailored. ${ }^{13}$ Moreover, natural polymers may not provide sufficient mechanical properties. ${ }^{14}$ Polyacrylic acid-based polymers are ideal candidates for drug delivery due to their swelling behavior in aqueous environments. ${ }^{15}$ These polymers are novel for $\mathrm{pH}$-sensitive drug delivery, which shows swelling at a higher $\mathrm{pH}$ due to the ionizable carboxyl groups, and can release drugs at a neutral $\mathrm{pH}$. One of the major disadvantages reported for a polyacrylic acid-based drug delivery system is rapid drug release, which can be controlled with cross-linking. Highly cross-linked systems provide slow drug release due to the small mesh size, which can be advantageous in drug delivery applications. The polymer also determines the physical properties of the hydrogel, including mechanical strength, degradability, and diffusivity of the releasing molecule. Dissolution of the polymer in an aqueous environment can be prevented with cross-linking. ${ }^{16}$

Curcumin, a naturally occurring yellow polyphenol from the rhizome of the perennial herb Curcuma longa, has potent anticancer properties. ${ }^{17}$ Curcumin inhibits proliferation as well as induces apoptotic cell death in numerous cell lines established from malignancies such as leukemia, breast, lung, prostate, and colon tumors. ${ }^{18-20}$ Studies in numerous carcinogenesis models ${ }^{21-25}$ and recent clinical trials in patients have confirmed the potential of curcumin as a tool for chemoprevention. However, clinical application is limited due to poor aqueous solubility, minimal systemic bioavailability, degradation at alkaline $\mathrm{pH}$, and photodegradation. The therapeutic index can be improved by entrapping curcumin in a biocompatible polymer, which can enhance solubility in an aqueous solution and extend the release.

However, hydrogel systems for hydrophobic drug delivery are rarely reported due to the incompatibility of the hydrophilicity of the polymer network and the hydrophobic drug. This problem can be solved by preparing semi-interpenetrating networks to tune the hydrophilicity to entrap hydrophobic drugs. In the current investigation, we developed a pH-sensitive, biodegradable, and biocompatible hydrogel to achieve site-specific drug delivery. We synthesized polyethylene glycol (PEG) cross-linked acrylic polymers and investigated the loading and release of curcumin. The method adopted for polymerizing the acrylic acid was the inverse emulsion polymerization method proposed by Vanderhoff et al in which an aqueous solution of a hydrophilic monomer, acrylic acid, is dispersed in a continuous lipophilic phase with the aid of surfactants to promote the formation of water in oil emulsion. ${ }^{26}$ Cell uptake and cytotoxicity studies were carried out in human cervical cancer cell lines (HeLa).

\section{Methods and materials \\ Materials}

Acrylic acid (molecular weight $=72$ ), cross-linked PEG diacrylate (molecular weight $=238)$, curcumin, ammonium persulfate, Span 80 (Sorbitan monooleate), Tween 80 (PEG sorbitan monooleate), and 3-(4,5-dimethylthiazol-2-yl)-2,5diphenyltetrazolium bromide (MTT) were purchased from Sigma-Aldrich (Steinheim, Germany). Immobilon Western reagent was purchased from Millipore (Billerica, MA). Rabbit polyclonal antibody against poly (ADP-ribose) polymerase (PARP) was obtained from Santa Cruz Biotechnology (Santa Cruz, CA). Rabbit monoclonal antibody against caspase- 3 and rabbit polyclonal antibody against caspase-9 were obtained from Cell Signaling (Beverly, MA). All other reagents and chemicals were of analytical grade or above, and used without further purification.

\section{Nanosphere preparation}

Nanoparticles were prepared with the inverse emulsion polymerization method. Emulsification was performed by dispersing the aqueous phase consisting of $10 \%$ acrylic acid, $5 \%$ sodium hydroxide, and $15 \%$ water with the continuous lipophilic phase consisting of liquid paraffin (68\%) and 
emulsifiers (2\%) and Span 80 and Tween 80 (75:25 ratio). One percent $\mathrm{PEG}$ diacrylate was added to the mixture as a cross-linker, and then the initiator, ammonium persulfate, was added. The temperature for polymerization was maintained at $60^{\circ} \mathrm{C}$ for 6 hours. The particles were isolated with centrifugation $(10,000 \mathrm{rpm})$ for 30 minutes. The isolated nanospheres were washed several times with hexane and then freeze-dried to remove the solvent and water. The freezedried products were stored in a vacuum. The polymerization of the acrylic acid with cross-linked PEG diacrylate was characterized with ${ }^{13} \mathrm{C}$ nuclear magnetic resonance spectroscopy (NMR) and Fourier transform infrared (FTIR) spectroscopy.

\section{Swelling studies}

The gravimetric method was used to study the swelling behavior of the cross-linked polymers as a function of $\mathrm{pH}$. A known quantity of the polymers was measured and immersed in an excess quantity of phosphate buffers with $\mathrm{pH}$ 2.2 and in phosphate buffered saline with $\mathrm{pH} 7.4$ for 24 hours to attain the swelling equilibrium. The weight of the swollen polymers was measured after excess solvent was removed. The swelling behavior was computed by calculating the percentage swelling $(\% \mathrm{~S})$ :

$$
\text { Percentage of swelling }=(\mathrm{Ws}-\mathrm{Wd}) / \mathrm{Wd} \times 100
$$

where Ws is the weight of the swollen polymer and $\mathrm{Wd}$ is the weight of the dried polymer.

\section{Drug loading}

Curcumin was loaded in polymer nanoparticles using the postpolymerization method. About $100 \mathrm{mg}$ of the lyophilized powder was dispersed in $10 \mathrm{~mL}$ of distilled water. Curcumin was dissolved in chloroform, and the drug solution in chloroform was added to the polymer solution with constant vortexing and sonication. The curcumin-loaded nanoparticles were then lyophilized to obtain dried powder.

\section{Entrapment efficiency}

A known amount of the lyophilized curcumin-loaded polymers was dissolved in methanol, vigorously vortexed to get a clear solution, and maintained for 24 hours, then filtered through a $0.1 \mu \mathrm{M}$ membrane filter. Absorbance of the filtrate was measured at $420 \mathrm{~nm}$ using LAMBDA 25 ultraviolet absorbance (PerkinElmer, Waltham, MA). The entrapped curcumin content was determined with the actual entrapment ratio (AER), expressed in terms of the amount of curcumin per the weight of the nanospheres. ${ }^{27}$ Entrapment efficiency can be calculated with the following equation:

$$
\text { Entrapment efficiency }(\%)=\text { AER/TER } \times 100
$$

where AER = measured drug wt/Nanosphere wt and theoretical entrapment ratio $($ TER $)=$ initial drug wt/drug wt and polymer wt, "nanogel wt" means the weight of the nanogel with curcumin measured to calculate the entrapment efficiency, and "initial drug wt" means the drug initially measured for the entrapment.

\section{Transmission electron microscopy}

Morphological analysis of the nanospheres was characterized with transmission electron microscopy (TEM; JEOL 1011, Tokyo, Japan). The samples of the nanoparticle suspension in Milli- $\mathrm{Q}^{\circledR}$ (Millipore Corporation, Billerica, MA) water at $25^{\circ} \mathrm{C}$ were dropped onto Formvar-coated grids, and measurements were taken only after the samples had completely dried.

\section{Differential scanning calorimetry}

Differential scanning calorimetry (DSC) was performed to analyze the state of the curcumin inside the polymer matrix. DSC thermograms of free curcumin and curcumin-loaded hydrogel were compared to analyze the physicochemical behavior of the nanoparticles. The DSC thermograms were obtained using an automatic thermal analyzer system (Pyris 6 DSC, PerkinElmer). Samples were crimped in standard aluminium pans and heated from $20^{\circ} \mathrm{C}$ to $250^{\circ} \mathrm{C}$ at a rate of $10^{\circ} \mathrm{C} /$ minute under constant purging of $\mathrm{N}_{2}$ at $10 \mathrm{~mL} /$ minute. An empty pan, sealed in the same way as the sample, was used as a reference.

\section{In vitro release kinetics}

A known amount of curcumin-loaded polyacrylic acid was dispersed in $10 \mathrm{~mL}$ phosphate buffer $(\mathrm{pH}$ 7.4) and was left in a shaking incubator at $37^{\circ} \mathrm{C} \pm 0.5^{\circ} \mathrm{C}$. At predetermined time intervals, a known quantity of the sample was withdrawn and replaced with fresh medium to maintain the total volume constant. The amount of curcumin released from the cross-linked hydrogel nanoparticles was measured with an ultraviolet spectrophotometer (PerkinElmer) at $420 \mathrm{~nm}$.

\section{Cell uptake studies}

Internalization of the curcumin-loaded hydrogel was studied in HeLa cells using confocal laser scanning microscopy that 
exploits the inherent fluorescence of curcumin (Leica TCS SP2, Leica Microsystems GmbH, Wetzlar, Germany). HeLa cells were grown in cover slips placed in 24 well plates, up to $80 \%$ confluency. Cells were then treated with $25 \mu \mathrm{M}$ free curcumin, curcumin-loaded hydrogels, and plain hydrogels. After 2 hours, the cells were washed, and the nuclei were stained with propidium iodide and examined under a confocal laser-scanning microscope for the intracellular fluorescence of curcumin.

\section{MTT assay}

Cytotoxicity studies of free curcumin and curcumin nanogels were carried out in HeLa cells using MTT dye assay. ${ }^{28} \mathrm{HeLa}$ cells were seeded $\left(3.0 \times 10^{3} /\right.$ well $)$ in a 96 -well culture plate and grown for 24 hours before the assay. The cells were then treated with different concentrations of free and curcumin nanogels $(5-50 \mu \mathrm{M})$ for 72 hours and then $20 \mu \mathrm{L}$ MTT $(5 \mathrm{mg} / \mathrm{mL})$ was added in $80 \mu \mathrm{L}$ culture medium (Dulbecco's modified Eagle's medium purchased from Invitrogen, Grand Island, NY, USA) to each well. After incubating for 2 hours at $37^{\circ} \mathrm{C}$, cells were lysed using lysis buffer, incubated for 1 hour, and the optical densities were measured at $570 \mathrm{~nm}$ using a microplate reader (Bio-Rad Laboratories, Hercules, CA). The relative cell viability in percentage was calculated as:

$\left(\mathrm{A}_{570}\right.$ of treated samples $/ \mathrm{A}_{570}$ of untreated samples $) \times 100(3)$

\section{Western blot analysis}

To detect the cleavage of caspases and PARP by curcumin/ curcumin nanogels, Western blot was performed as previously described. ${ }^{28}$ Briefly, $0.6 \times 10^{6}$ cells were plated in $60 \mathrm{~mm}$ plates and treated with curcumin for 24 hours. The total protein isolated was resolved in a sodium dodecyl sulfatepolyacrylamide gel electrophoresis, immunoblotted using anticaspase-3, anticaspase-9, and anti-PARP antibody, and detected with enhanced chemiluminescence.

\section{Clonogenic assay}

The cells were seeded in six-well plates and treated with curcumin/curcumin nanogels for 72 hours after which the clonogenic assay was performed as previously described. ${ }^{29}$ Then $10^{4}$ cells in six-well plates were treated with curcumin for 72 hours. Following this, fresh medium was added and incubated for 1 week. The clones developed were fixed in glutaraldehyde and stained using crystal violet. A colony containing more than four cells was counted as one clone. The clones were counted and compared with those of the untreated control.

\section{Fluorescence activated cell sorting}

The cells were subjected to curcumin/curcumin nanogel $(25 \mu \mathrm{M})$ treatment for 24 hours and analyzed with fluorescence activated cell sorting (FACS) as described previously. ${ }^{30}$ Briefly, the cells were trypsinized, and the cell pellets were resuspended in $70 \%$ cold ethanol and incubated for 30 minutes at $4{ }^{\circ} \mathrm{C}$. The permeabilized cells were spun down and suspended in $250 \mu \mathrm{L}$ of phosphate buffered saline. To this, $5 \mu \mathrm{L}$ of RNase A $(10 \mathrm{mg} / \mathrm{mL})$ was added, incubated at $37^{\circ} \mathrm{C}$ for 30 minutes, stained with $10 \mu \mathrm{L}$ of propidium iodide $(1 \mathrm{mg} / \mathrm{mL})$, and analyzed using the FACS Aria flow cytometer (BD Biosciences, Franklin Lakes, NJ).

\section{Results}

\section{Characterization of nanogels}

Curcumin nanogels in the cross-linked polymer combinations $(0.5 \%$ and $1 \%)$ were successfully prepared using the inverse emulsion polymerization method (Figure 1). The particles' morphology and size were studied using TEM. The nanoparticles obtained ranged from 50 to $100 \mathrm{~nm}$ with a narrow size distribution. Cross-linking was confirmed with FTIR spectroscopy (Figure 2) and ${ }^{13} \mathrm{C}$ NMR (Figure 3).

The FTIR spectrum shows an absorption band at $1721 \mathrm{~cm}^{-1}$ due to the $\mathrm{C}=\mathrm{O}$ of PEG diacrylate and at $2926 \mathrm{~cm}^{-1}$ due to asymmetrical stretching of the methylene groups. The peaks at $3556 \mathrm{~cm}^{-1}$ and $1038 \mathrm{~cm}^{-1}$ were observed due to the $-\mathrm{OH}$ and $-\mathrm{COC}$ stretching frequencies. Furthermore, absorption peaks at $1560 \mathrm{~cm}^{-1}$ and $1404 \mathrm{~cm}^{-1}$ were assigned to asymmetric and symmetric stretching vibration of the carboxylate ion.

${ }^{13} \mathrm{C}$ NMR showed a peak at around $39 \mathrm{ppm}$, which corresponds to the $\mathrm{C}-\mathrm{C}$ bond, and around $180 \mathrm{ppm}$, which corresponds to the peak for the $\mathrm{C}=\mathrm{O}$ bond. The peak at $61 \mathrm{ppm}$ and the peak at $63 \mathrm{ppm}$ are attributed to the chemical shift of the $\mathrm{C}-\mathrm{O}$ and $-\mathrm{OH}$ bonds, respectively. The peak at 70 ppm shows the $\mathrm{CH}_{2}$ of PEG diacrylate.

The degree of cross-linking, variation in particle size, and encapsulation efficiency are summarized in Table 1. The entrapment efficiency of the $0.5 \%$ and $1 \%$ cross-linked polymers was $71.6 \% \pm 1.6 \%$ and $67.5 \% \pm 0.51 \%$, respectively. Results showed the entrapment efficiency decreased as cross-linking increased, which might be due to differences in the swelling behavior.

The influence of $\mathrm{pH}$ on the nanogels' swelling capacity was also analyzed (Table 1). The effect of $\mathrm{pH}$ on the swelling of the polymers was studied with gravimetric analysis. Sample A (0.5\%) showed swelling of $36.6 \pm 0.82 \mathrm{~g} / \mathrm{g}$ of nanogel at $\mathrm{pH} 7.4$ and $16.4 \pm 0.72 \mathrm{~g} / \mathrm{g}$ of nanogel at $\mathrm{pH}$ 2.2 whereas B (1\%) showed swelling of $21.2 \pm 1.34 \mathrm{~g} / \mathrm{g}$ of 


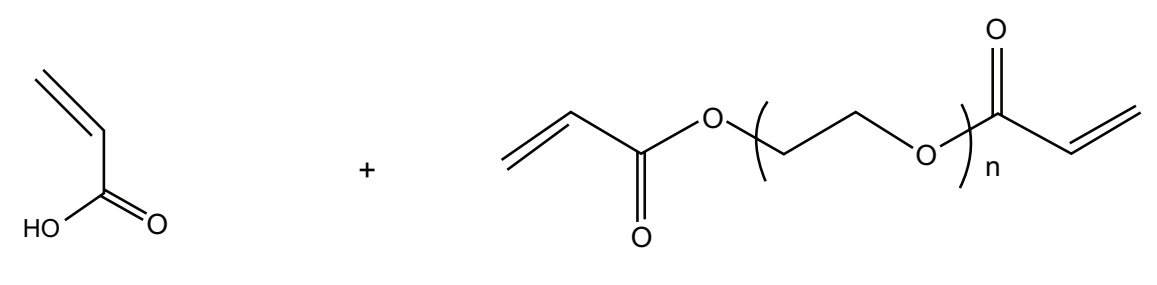

Acrylic acid

Polyethylene (glycol) diacrylate

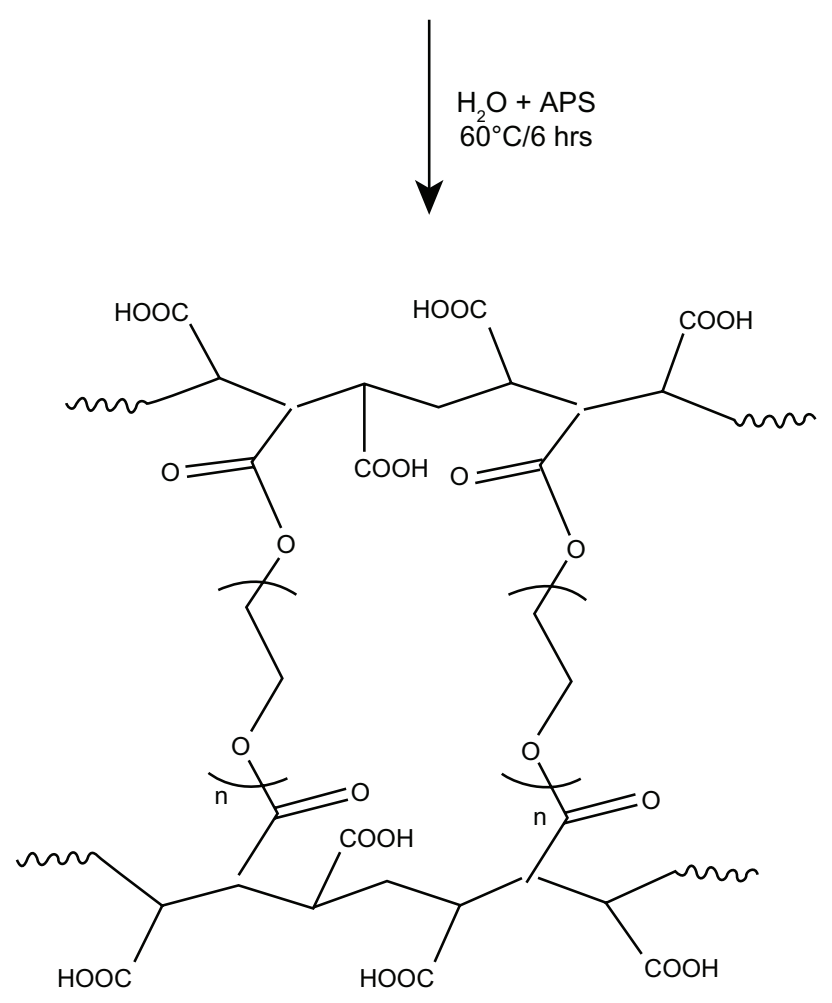

Figure I Synthesis of polyethylene glycol cross-linked acrylic polymers.

Abbreviation: APS, ammonium persulfate.

nanogel and $17.8 \pm 1.22 \mathrm{~g} / \mathrm{g}$ of nanogel at $\mathrm{pH} 7.4$ and $2.2, \quad$ In vitro drug release and cellular uptake respectively.

TEM images showed the mean diameter of the particles with $0.5 \%$ and $1 \%$ cross-linking was 50 and $100 \mathrm{~nm}$, respectively (Figure 4A and B). Particle size increased when cross-linking increased.

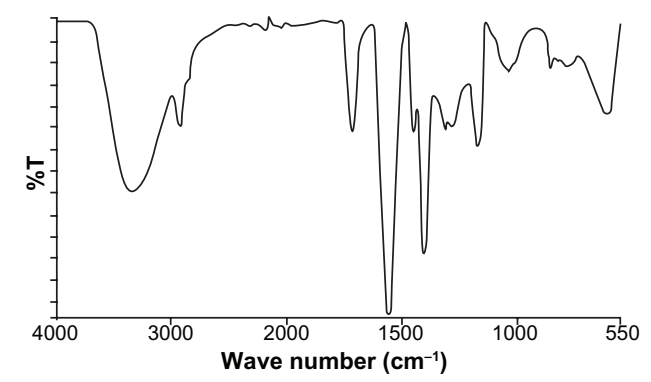

Figure 2 Fourier transform infrared spectra of polyethylene glycol cross-linked acrylic polymers.
Free curcumin is poorly soluble in aqueous medium and forms flakes but readily soluble in dimethyl sulfoxide (DMSO). This makes it very difficult to administer curcumin for in vivo studies, as DMSO is a highly toxic chemical and cannot be used in vivo. The in vitro release profile of

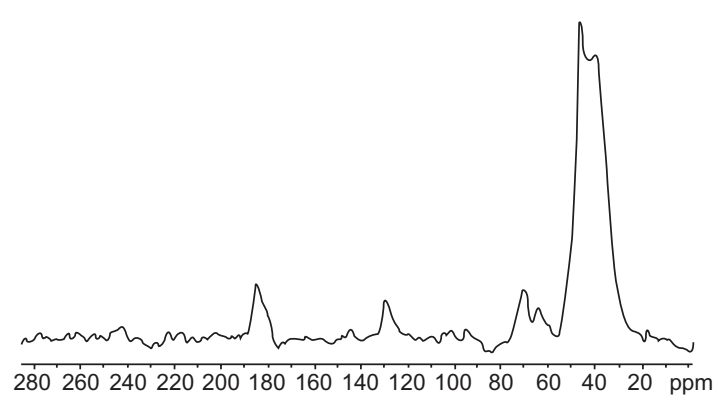

Figure $3{ }^{13} \mathrm{C}$ nuclear magnetic resonance spectrum of acrylic polymers. 
Table I Degree of swelling, entrapment efficiency, and particle size of hydrogels

\begin{tabular}{llllll}
\hline Sample & $\begin{array}{l}\text { Degree of } \\
\text { cross-linking }\end{array}$ & $\begin{array}{l}\text { Entrapment } \\
\text { efficiency }(\%)\end{array}$ & $\begin{array}{l}\text { Particle } \\
\text { size }(\mathbf{n m})\end{array}$ & $\begin{array}{l}\text { Swelling behavior at } \\
\mathbf{p H ~ 7 . 4}(\mathbf{g} / \mathbf{g} \text { of } \text { nanogel) }\end{array}$ & $\begin{array}{l}\text { Swelling behavior at } \\
\mathbf{p H ~ 2 . 2}(\mathbf{g} / \mathbf{g} \text { of } \mathbf{n a n o g e l})\end{array}$ \\
\hline $\mathrm{A}$ & $0.5 \mathrm{~mol} \%$ & $71.6 \pm 1.6$ & $50 \mathrm{~nm}$ & $36.6 \pm 0.82$ & $16.4 \pm 0.72$ \\
B & $1.0 \mathrm{~mol} \%$ & $67.5 \pm 0.5 \mathrm{I}$ & $100 \mathrm{~nm}$ & $21.2 \pm 1.34$ & $17.8 \pm 1.22$ \\
\hline
\end{tabular}

the entrapped anticancer drug curcumin from two different cross-linked polymer systems is shown in Figure 5. In the case of the $0.5 \%$ cross-linked polymers, there was an initial burst release of $40 \%$ and complete release occurred in 4 hours due to the swelling of the nanogels at $\mathrm{pH} 7.4$ and the increase in the mesh size, which might make it difficult to control the diffusion of curcumin. However, in the case of the $1 \%$ cross-linked polymers, there was an initial release of around $12 \%$ and thereafter the release occurred in a sustained manner, which might be due to the decrease in the swelling because of the increase in cross-linking. Curcumin exhibits green fluorescence after exhibiting excitation at
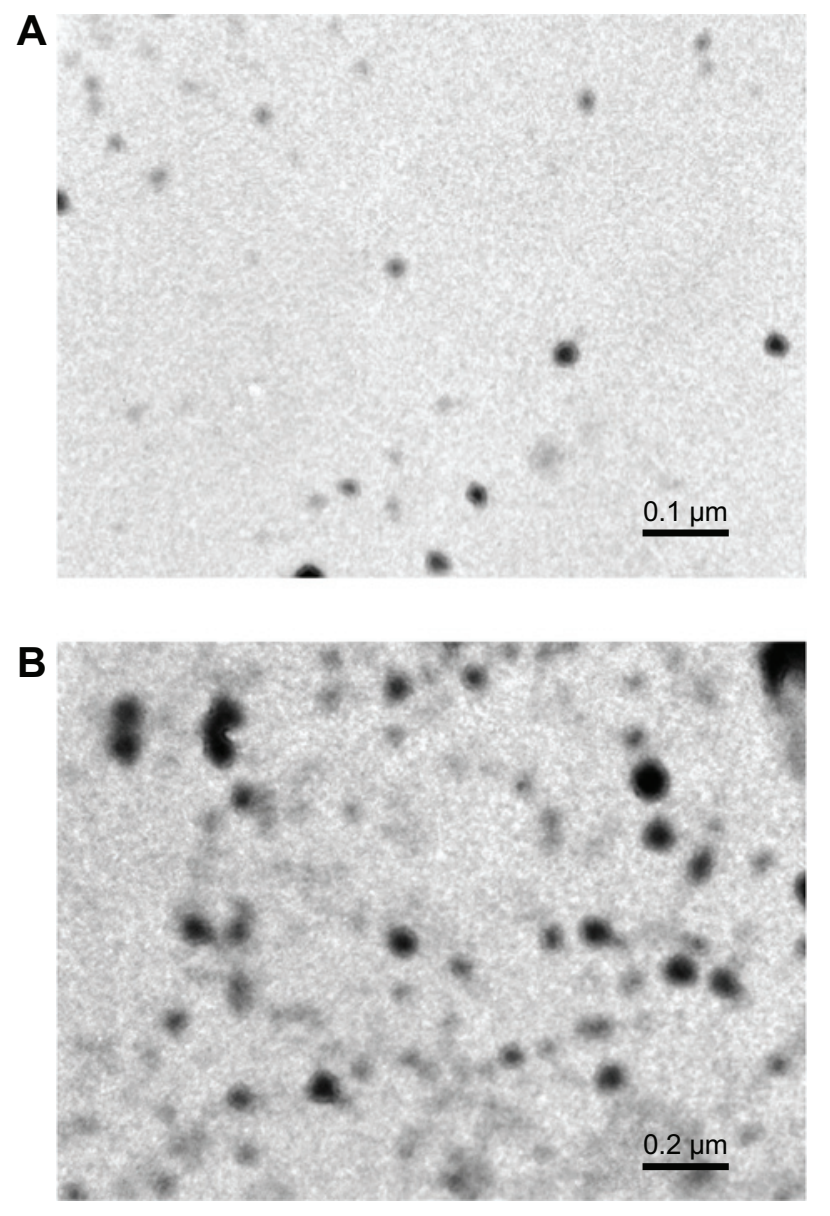

Figure 4 Transmission electron micrographs of polyethylene glycol diacrylate crosslinked acrylic polymers with (A) $0.5 \%$ cross-linking and (B) $1.0 \%$ cross-linking. Note: Particle size increased as cross-linking increased.
$420 \mathrm{~nm}$. We observed a strong fluorescence in cells treated with curcumin nanogels suspended in medium and curcumin dissolved in DMSO, indicating the cellular uptake of curcumin nanogels is equivalent to that of curcumin dissolved in DMSO (Figure 6).

\section{Significant cytotoxicity exhibited by the nanogel form of curcumin-encapsulated I\% cross-linked polymers}

MTT assay was used to compare the cytotoxicity of the curcumin-encapsulated polymers suspended in aqueous medium with that of curcumin dissolved in DMSO. The cytotoxicity of curcumin in DMSO was compared with that of curcuminencapsulated cross-linked polymers in HeLa cells (Figure 7). The curcumin nanogels with $1 \%$ cross-linked polymers showed slightly more cytotoxicity compared with the $0.5 \%$ cross-linked polymers and curcumin in DMSO especially at the half-maximal inhibitory concentration $\left(\mathrm{IC}_{50}\right)$ range while the blank polymers showed no significant toxicity.

\section{Better apoptosis of curcumin nanogels compared with curcumin in DMSO}

The ability of curcumin nanogels to induce apoptosis in HeLa cells was evaluated by the extent of the cleavage

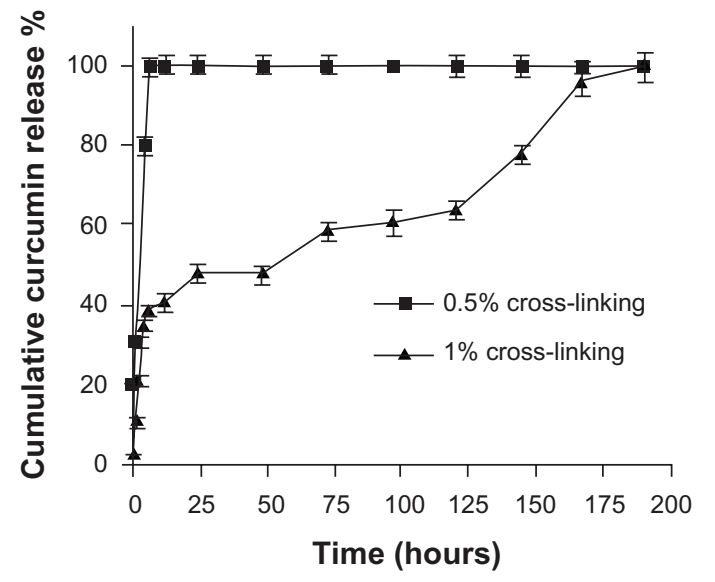

Figure 5 In vitro release of curcumin from polyethylene glycol diacrylate crosslinked acrylic hydrogels with two different percentages of cross-linking: hydrogels with $1.0 \%$ cross-linking showed slower release than those with $0.5 \%$ cross-linking. 


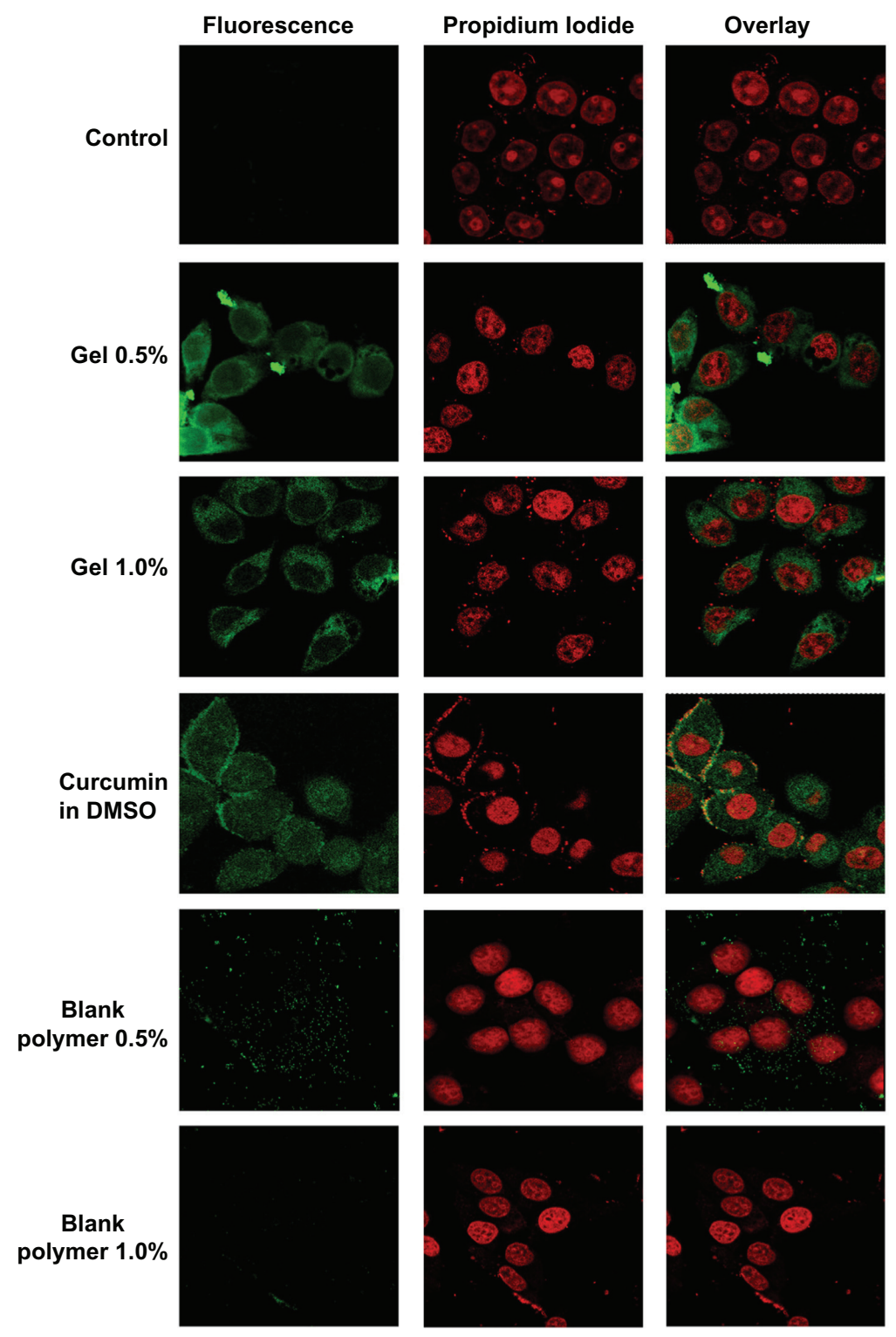

Figure 6 Cellular uptake of curcumin and curcumin nanogels: Human cervical cancer cell lines were treated with $25 \mu \mathrm{M}$ of curcumin in dimethyl sulfoxide, curcumin in medium, and hydrogels with $0.5 \%$ cross-linking or $1 \%$ cross-linking, and confocal images were taken after excitation at $420 \mathrm{~nm}$.

of the initiator caspase-9, the effector caspase-3, and the DNA-repairing protein, PARP, the downstream substrate of caspase-3 and a hallmark of apoptosis, ${ }^{31}$ and compared with that of free curcumin in DMSO, which has been reported by our group. ${ }^{32}$ The $0.5 \%$ and $1 \%$ curcumin nanogels cleaved the caspases- 3 and -9 more effectively than free curcumin in DMSO (Figure 8A and B). Similarly, when the cells were treated with 10 and $25 \mu \mathrm{M}$ curcumin, the nanogel forms induced PARP cleavage, and the curcumin nanogels with $1 \%$ cross-link showed better efficacy compared with curcumin in DMSO (Figure 8C). In the cells treated with curcumin nanogels with $1 \%$ cross-link, the $116 \mathrm{kDa}$ form was almost completely degraded to the cleaved $85 \mathrm{kDa}$ form, which was further degraded into smaller fragments, indicating the extent of caspase- 3 activation induced by these polymers was much higher than that of the $0.5 \%$ polymers and curcumin in DMSO.

\section{Downregulation of clonogenic potential of HeLa cells by curcumin nanogels compared with that of curcumin in DMSO}

Using clonogenic assay, we investigated whether curcumin or curcumin nanogels with different percentages of crosslinking can downregulate the clonogenic potential of $\mathrm{HeLa}$ 


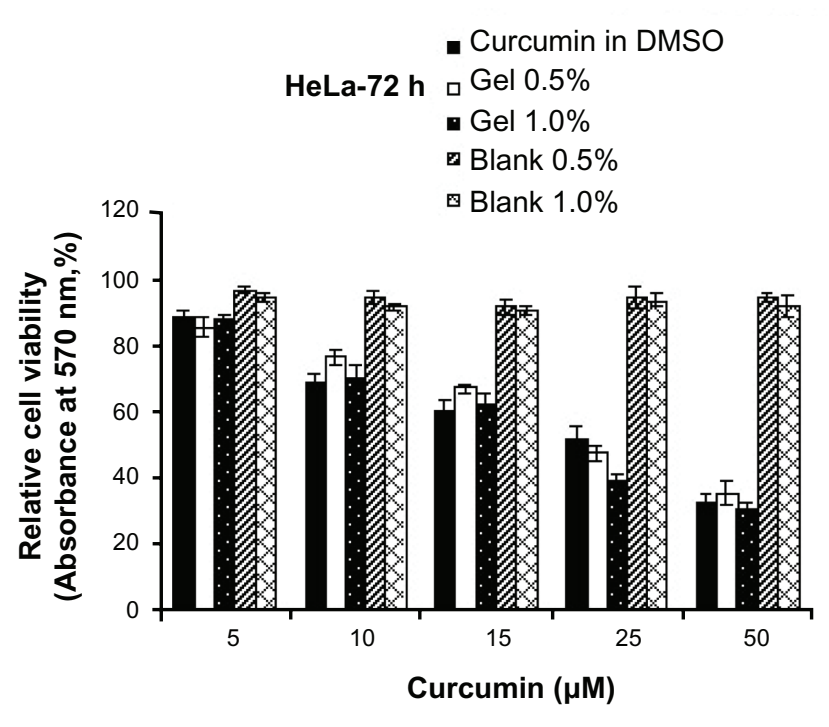

Figure $7 \mathrm{Gel}$ form of curcumin is as effective or slightly more effective in inducing cytotoxicity: (4,5-dimethylthiazol-2-yl)-2, 5-diphenyltetrazolium bromide assay was conducted in human cervical cancer cell lines at various concentrations and time intervals as indicated.

Abbreviations: HeLa, human cervical cancer cell lines; DMSO, dimethyl sulfoxide.

cells. The results clearly indicate the clonogenic potential of HeLa cells was significantly inhibited by the curcumin nanogels $(P<0.001)$ and by curcumin in DMSO (Figure 9). The inhibition of clonogenic potential by curcumin nanogels was slightly higher compared with curcumin in DMSO.

A

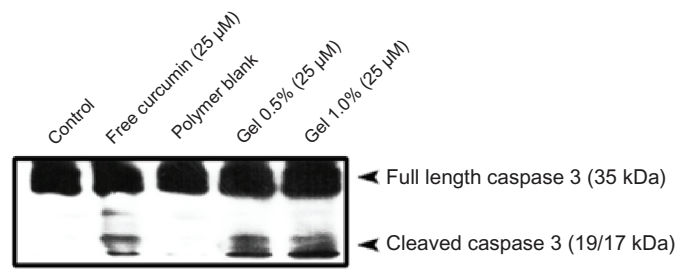

B

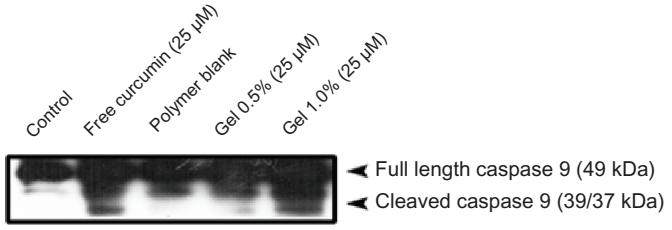

C

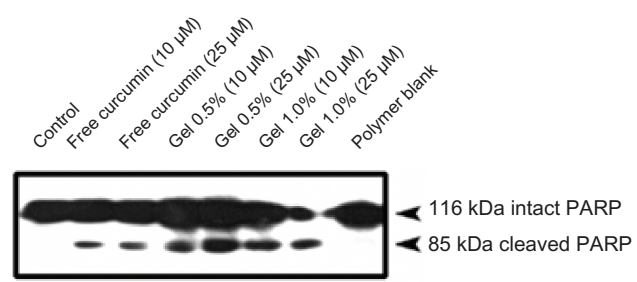

Figure 8 Curcumin nanogels enhance poly(ADP-ribose) polymerase cleavage in human cervical cancer cell lines: Human cervical cancer cell lines cells were treated with the indicated concentrations of free curcumin/curcumin nanogels for 24 hours, and the entire cell lysate was immunoblotted against (A) anticaspase-3, (B) anticaspase-9, and (C) antipoly(ADP-ribose) polymerase antibodies.

\section{The nanogel forms of curcumin induce more $\mathrm{G} 2 / \mathrm{M}$ arrest compared to curcumin in DMSO}

Cell cycle arrest induced by curcumin in HeLa cells was also examined. The results clearly indicate that curcumin/curcumin nanogels induce $\mathrm{G} 2 / \mathrm{M}$ arrest in HeLa cells. While curcumin in DMSO induced $28 \% \mathrm{G} 2 / \mathrm{M}$ arrest, the $0.5 \%$ and $1 \%$ curcumin nanogels induced $19.5 \%$ and $24.7 \%$ G2/M arrest, respectively. Although not much difference was observed in the cell cycle arrest between the various forms of curcumin, the $1 \%$ curcumin nanogels $(1.0 \%)$ were slightly better than the $0.5 \%$ curcumin nanogels, as shown in Figure 10.

\section{Discussion}

Curcumin, a yellow pigment present in the spice turmeric (Curcuma longa), has been linked with antioxidant, antiinflammatory, antiproliferative, anticancer, antidiabetic, antirheumatic, and antiviral effects, but the phytochemical's optimum potential is limited by a lack of solubility in aqueous solvents and poor oral bioavailability, which limit its use as a potential chemotherapeutic drug. ${ }^{33}$ Nanoparticle-mediated drug delivery sytems have proved excellent solutions to these problems. ${ }^{34}$

Inverse emulsion polymerization is a controllable method for preparing well-defined nanoparticles. In this study, we prepared acrylic polymers cross-linked with two different molecular weight percentages of PEG diacrylate using the inverse microemulsion polymerization method (the schematic representation is given in Figure 11). This method facilitates free radical polymerization of an acrylic monomer with PEG diacrylate in the presence of ammonium persulfate. PEG is a highly biocompatible and hydrophilic polymer with a low glass transition temperature. Including polyacrylic acid in the system imparts $\mathrm{pH}$ sensitivity. The nanogels we prepared also displayed a $\mathrm{pH}$-sensitive nature, which can be exploited for site-specific controlled drug delivery.

Cross-linking was confirmed with FTIR spectroscopy (Figure 2) and ${ }^{13} \mathrm{C}$ NMR (Figure 3 ). The effect of size on the biological performance of nanoparticles is well documented; an optimal diameter range is believed to favor prolonged circulation times and passive targeting of carriers thanks to the enhanced permeation retention effect. ${ }^{35}$ Below $200 \mathrm{~nm}$ and above $10-20 \mathrm{~nm}$ is the optimum size for the enhanced permeation retention effect. ${ }^{36}$ TEM images showed that the mean diameter of the particles with $1 \%$ cross-linking was greater than that of the particles with $0.5 \%$ cross-linking, which shows nanogels 


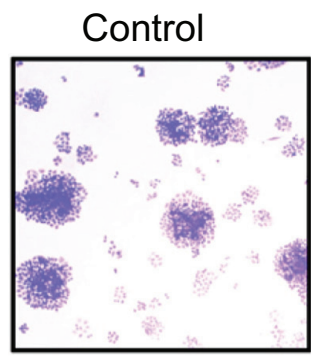

Curcumin DMSO
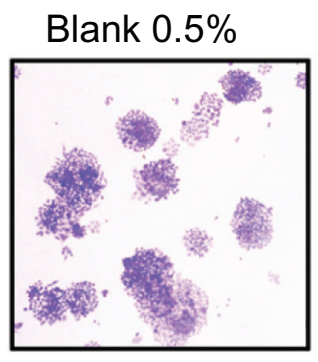

Blank 1.0\%

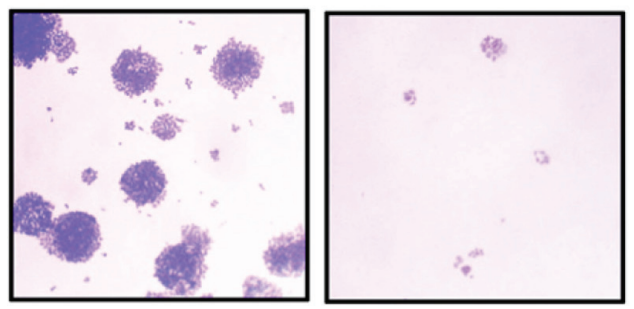

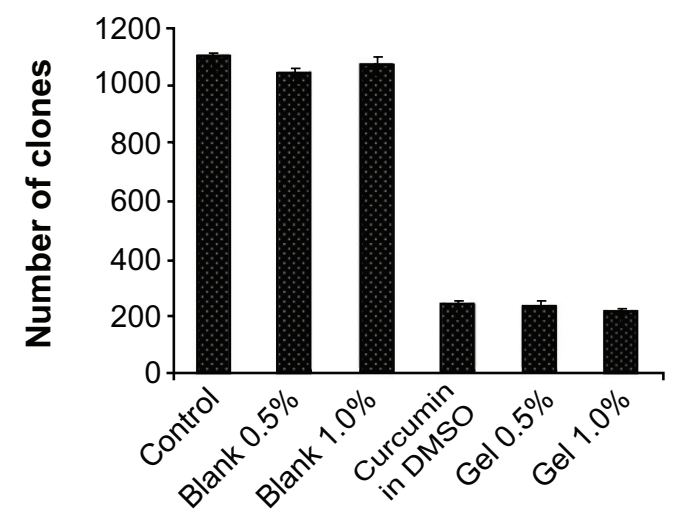

Figure 9 Curcumin and curcumin nanogels inhibit the clonogenic potential of human cervical cancer cell lines: Human cervical cancer cell lines were treated with curcumin dissolved in dimethyl sulfoxide or curcumin nanogels for 72 hours, fresh medium was added and maintained for I week, and the clones developed were stained and counted. Abbreviation: DMSO, dimethyl sulfoxide.

with $1 \%$ cross-linked polymers have better encapsulation efficiency - one of the reasons for better anticancer activity. Particle size increased as cross-linking increased. DSC of the PEG diacrylate cross-linked acrylic polymers indicates an endothermic transition at $120^{\circ} \mathrm{C}$ that can be attributed to the loss of loose and bound water in the hydrogel. The nanogel appeared to be thermally stable up to $200^{\circ} \mathrm{C}$. Curcumin was loaded onto the cross-linked acrylic polymers through physical adsorption using the postloading method. Cross-linking using PEG diacrylate provides hydrophobicity to the acrylic hydrogel, which enhances the uptake of curcumin, a hydrophobic drug. Results showed that entrapment efficiency decreased when cross-linking increased, which might be due to differences in the swelling behavior (Table 1). Swelling studies revealed that $\mathrm{pH}$ and cross-linking influence polymer swelling. Polymer swelling occurs at a $\mathrm{pH}$ above the $\mathrm{pKa}$ of the carboxyl group of acrylic acids. Swelling increases as the COO concentration increases, which can be reduced by enhancing the cross-linking density.

The in vitro release kinetic profile followed a biphasic pattern: an initial burst release followed by a sustained release (Figure 5). The burst release of the curcumin from the polymers was controlled by increasing the cross-linking. Hydrogels with $1 \%$ cross-linked polymers showed an initial release of around $12 \%$, and thereafter the release occurred in a sustained manner, which might be due to the decrease in the swelling because of the increase in cross-linking. Controlled delivery by cross-linked polymers is a desirable property for drug delivery applications.

To biologically evaluate curcumin nanogels, we compared the intracellular fluorescence of curcumin nanogels dispersed in aqueous medium with that of free curcumin in DMSO. The confocal images of the cell uptake studies show curcumin nanogels have almost similar cellular uptake, in vitro, as their free counterpart (Figure 6). These data confirm curcumin nanogels overcome the barrier of curcumin's aqueous dispersibility, which facilitates administration in vivo, by avoiding use of toxic solvents like DMSO.

We observed that curcumin nanogels show more cytotoxicity and apoptotic effects toward cancer cells than free curcumin, as assessed by cleavage of caspases and their downstream target, PARP, in HeLa cells. Previous studies 

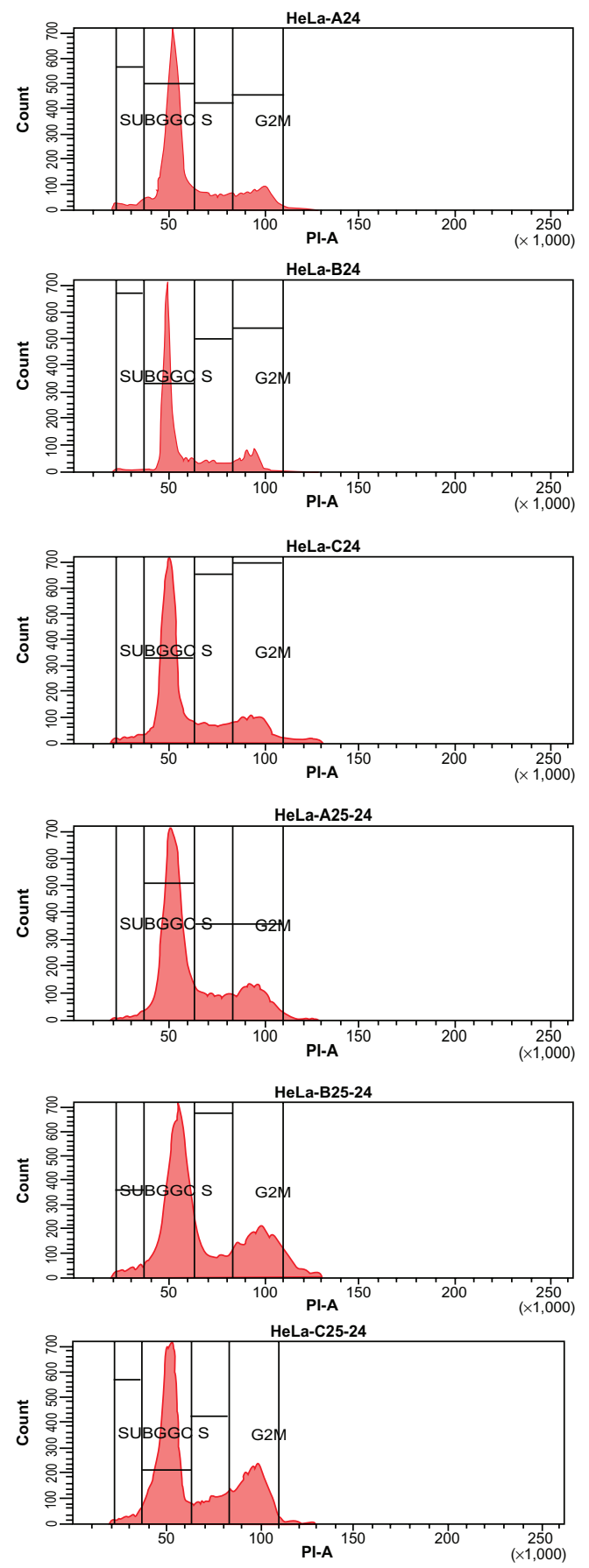

\begin{tabular}{|lrrr|}
\hline Tube: A24 & & & \\
Population & \# events & $\%$ parent & $\%$ total \\
\hline All events & 13,093 & & 100.0 \\
& 9,763 & 74.6 & 74.6 \\
\hline G1 & 6,151 & 63.0 & 47.0 \\
S & 1,287 & 13.2 & 74.6 \\
G & 1,717 & 17.6 & 13.1 \\
\hline
\end{tabular}

\begin{tabular}{|lrrr|}
\hline Tube: B24 & & & \\
Population & \# events & $\%$ parent & $\%$ total \\
\hline All events & 8,279 & & 100.0 \\
\hline P1 & 6,524 & 78.8 & 78.8 \\
\hline G1 & 4,323 & 66.3 & 52.2 \\
S G2M & 864 & 13.2 & 10.4 \\
X SUB GO & 1,167 & 17.9 & 14.1 \\
\hline
\end{tabular}

\begin{tabular}{|lrrr|}
\hline Tube: C24 & & & \\
Population & \# events & $\%$ parent & $\%$ total \\
\hline All events & 26,471 & & 100.0 \\
\hline P1 & 19,130 & 72.3 & 72.3 \\
\hline S1 & 12,374 & 64.7 & 46.7 \\
\hline G2M & 2,407 & 12.6 & 9.1 \\
SUB GO & 3,390 & 17.7 & 12.8 \\
\hline
\end{tabular}

\begin{tabular}{|lrrr|}
\hline Tube: A25-24 & & & \\
Population & \# events & $\%$ parent & $\%$ total \\
\hline All events & 27,984 & & 100.0 \\
& 21,107 & 75.4 & 75.4 \\
P1 & 12,912 & 61.2 & 46.1 \\
S 1 & 3,082 & 14.6 & 11.0 \\
S G2M & 4,113 & 19.5 & 14.7 \\
SUB GO & 483 & 23 & 1.7 \\
\hline
\end{tabular}

\begin{tabular}{|lrrr|}
\hline Tube: B25-24 & & & \\
Population & \# events & $\%$ parent & $\%$ total \\
\hline All events & 29,513 & & 100.0 \\
& 19,910 & 67.5 & 67.5 \\
P1 & 10,860 & 54.5 & 36.8 \\
& 2,479 & 12.5 & 8.4 \\
S 1 & 4,912 & 24.7 & 16.6 \\
S GM & 568 & 2.9 & 1.9 \\
\hline
\end{tabular}

\begin{tabular}{|lrrr|}
\hline Tube: C25-24 & & & \\
Population & \# events & $\%$ parent & $\%$ total \\
\hline All events & 27,473 & & 100.0 \\
P1 & 19,363 & 70.5 & 70.5 \\
\hline G1 & 10,436 & 53.9 & 38.0 \\
S & 2,458 & 12.7 & 8.9 \\
G2M & 5,431 & 28.0 & 19.8 \\
SUB GO & 577 & 3.0 & 2.1 \\
\hline
\end{tabular}

Figure 10 Curcumin and nanogel curcumin induce G2/M arrest of human cervical cancer cell lines.

Notes: Nanogel I.0\% is slightly better than nanogel 0.5\%. A24: Blank 0.5\%; B24: Blank 1.0\%; C24: Control; A25-24: Curcumin nanogel 0.5\%; B25-24: Curcumin nanogel I.0\%; C25-24: Curcumin in DMSO. Curcumin concentration is $25 \mu \mathrm{M}$.

Abbreviation: DMSO, dimethyl sulfoxide.

indicate that nanoencapsulation of curcumin can downregulate the anchorage-independent proliferation of tumor cells. ${ }^{37} \mathrm{We}$ observed a marked difference in the number and size of the clones between curcumin nanogels and the untreated control, which is in concordance with the results of the cytotoxicity study. Inhibition of colony formation by curcumin nanogels is as effective as free curcumin, which supports the idea of using curcumin nanogels for sustained anticancer activity. We observed the curcumin-adsorbed acrylic nanogels are water soluble and as effective as or more active than free curcumin as assessed with viability assays as well as Western blotting, clonogenic assay, and FACS analysis.

Drugs can be entrapped and released from hydrogel nanoparticles due to the good swelling, biodegradability, 


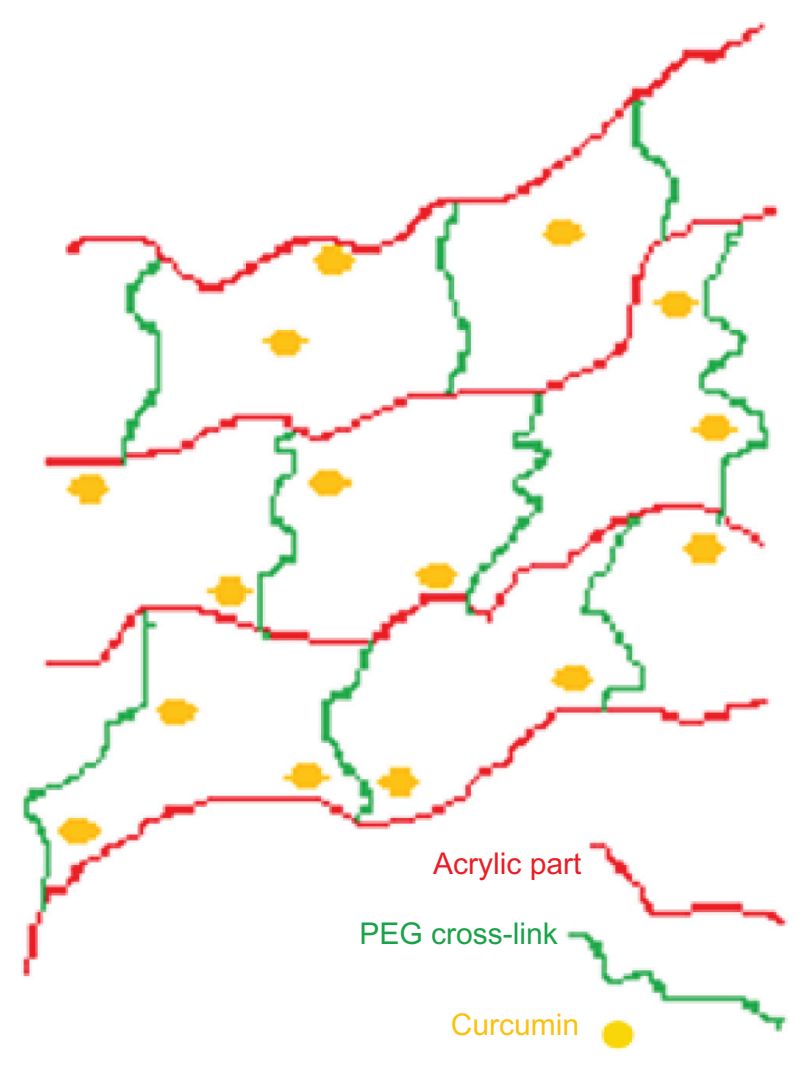

Figure II Schematic representation of polyethylene glycol cross-linked acrylic hydrogels.

and size distribution properties. The in vitro release rate of curcumin showed the sustained release from hydrogels with increased cross-linking, and the releasing rate was also dependent on the $\mathrm{pH}$ of the releasing medium. Although further studies are required to prove the efficacy of this formulation in vivo, successful implementation of curcumin nanogels with some modifications may prove very advantageous to assign curcumin as a chemotherapeutic rather than a chemopreventive agent.

\section{Conclusion}

Biocompatible, ph-sensitive, and biodegradable hydrogels as novel drug delivery systems were developed to achieve localized drug delivery. The inverse microemulsion polymerization method was used to synthesize PEG cross-linked acrylic polymers and the loading and release of curcumin was investigated. Intracellular and cytotoxicity studies were carried out in HeLa cell lines. A possible cause for higher anticancer activity could be higher cellular uptake of curcumin nanogels. Encapsulation of curcumin within cross-linked hydrogels shows comprehensive results, justifying the potential use of curcumin nanogels with better solubility, greater cellular uptake, sustained release, and enhanced antiproliferative effect to induce apoptosis in aqueous medium compared with free curcumin in DMSO. Overall, the results suggest curcumin nanogels are likely to have great potential as therapeutic agents.

\section{Acknowledgments}

The authors thank the Department of Biotechnology, Government of India, for financial support and the Sophisticated Analytical Instruments Facility (SAIF) Kerala for carrying out IR, DSC, and NMR.

\section{Disclosure}

The authors report no conflicts of interest in this work.

\section{References}

1. Kim IS, Jeong YI, Kim DH, Lee YH, Sung-Ho Kim SH. Albumin release from biodegradable hydrogels composed of dextran and poly(ethylene glycol) macromer. Arch Pharm Res. 2001;24:69-73.

2. Peppas NA, Khare AR. Preparation, structure and diffusional behavior of hydrogels in controlled release. Adv Drug Deliv Rev. 1993;11:1-35.

3. Peppas NA, Bures P, Leobandung W, Ichikawa H. Hydrogels in pharmaceutical formulations. Eur J Pharm Biopharm. 2000;50:27-46.

4. Brazel CS, Peppas NA. Mechanisms of solute and drug transport in relaxing, swellable, hydrophilic glassy polymers. Polymer. 1999;40: 3383-3398.

5. Siegal RA, Firestone BNA. PH-Dependent equilibrium swelling properties of hydrophobic polyelectrolyte copolymer gels. Macromolecules. 1988;21:3254-3259.

6. Nair KL, Jagadeeshan S, Nair SA, Kumar GSV. Biological evaluation of 5-fluorouracil nanoparticles for cancer chemotherapy and its dependence on the carrier, PLGA. Int J Nanomedicine. 2011;6:1685-1697.

7. Nair KL, Jagadeeshan S, Nair SA, Kumar GSV. Evaluation of triblock copolymeric micelles of $\delta$-Valerolactone and poly(ethylene glycol) as a competent vector for doxorubicin delivery against cancer. J Nanobiotechno. 2011;9:42.

8. Gander B, Gurry R, Doelker, Peppas NA. Effect of polymeric network structure on drug release from crosslinked poly (vinyl alcohol) micromatrices. Pharm Res. 1989;6:578-584.

9. Colombo P, Santi P, Bettini R, Brazel CS. Drug release from swelling-controlled systems. In: Wise D, editor. Handbook of Pharmaceutical Controlled Release Technology. New York: Marcel Dekker; 2000:183-209.

10. Brannon-Peppas, L. Recent advances in the use of biodegradable microparticles and nanoparticles in controlled drug delivery. Int J Pharm. 1995;116:1-9.

11. Kost J, Langer R. Equilibrium swollen hydrogels in controlled release applications. In: Peppas NA, editor. Hydrogels in Medicine and Pharmacy. Boca Raton: CRC Press; 1986;3:95-108.

12. Kim B, La Flamme K, Peppas NA. Dynamic swelling behavior of $\mathrm{pH}$-sensitive anionic hydrogels uses for protein delivery. $J$ Appl Polym Sci. 2003;89:1606-1613.

13. Khare AR, Peppas NA. Swelling/deswelling of anionic copolymer gels. Biomaterials. 1995; 16:559-567.

14. Lin CC, Metters AT. Hydrogels in controlled release formulations: network design and mathematical modelling. Adv Drug Delivery Rev. 2006;58:1379-1408.

15. Burugapalli K, Bhatia D, Koul V, Choudhary V. Interpenetrating polymer networks based on poly(acrylic acid) and gelatin I: swelling and thermal behavior. J Appl Polym Sci. 2001;82:217-227.

16. Hennick WE, van Nostrum CF. Novel cross linking methods to design hydrogels. Adv Drug Delivery Rev. 2002;54:13-26. 
17. Goel A, Kunnumakkara AB, Aggarwal BB. Curcumin as "Curecumin": from kitchen to clinic. Biochem Pharmacol. 2008;75:787-809.

18. Shishodia S, Sethi G, Aggarwal BB. Curcumin: getting back to the roots. Ann NY Acad Sci. 2005;1056:206-217.

19. Strimpakos AS, Sharma RA. Curcumin: preventive and therapeutic properties in laboratory studies and clinical trials. Antioxid Redox Sign. 2008; 10:511-546.

20. Anand P, Sundaram C, Jhurani S, Kunnumakkara AB, Aggarwal BB. Curcumin and cancer: an "old-age" disease with an "age-old" solution. Cancer Lett. 2008;267:133-164.

21. Chuang SE, Kuo ML, Hsu CH, et al. Curcumin-containing diet inhibits diethylnitrosamine-induced murine hepatocarcinogenesis. Carcinogenesis. 2000;21:331.

22. Inano $\mathrm{H}$, Onoda $\mathrm{M}$, Inafuku $\mathrm{N}$, et al. Chemoprevention by curcumin during the promotion stage of tumorigenesis of mammary gland in rats irradiated with \{gamma\}-rays. Carcinogenesis. 1999;20:1011.

23. Kawamori T, Lubet R, Steele VE, et al. Chemopreventive effect of curcumin, a naturally occurring anti-inflammatory agent, during the promotion/progression stages of colon cancer. Cancer Res. 1999;59:597.

24. Li N, Chen X, Liao J, et al. Inhibition of 7,12-dimethylbenz[a]anthracene (DMBA)-induced oral carcinogenesis in hamsters by tea and curcumin. Carcinogenesis. 2002;23:1307.

25. Singh SV, Hu X, Srivastava SK, et al. Mechanism of inhibition of benzo[a]pyrene-induced forestomach cancer in mice by dietary curcumin. Carcinogenesis. 1998;19:1357.

26. Vanderhoff JW, Branford EB, Tarkowski HL. Inverse emulsion polymerization. Adv Chem Ser. 1962;34:32-51.

27. Qiu Y, Park K. Environment-sensitive hydrogels for drug delivery. $A d v$ Drug Delivery Rev. 2001;53:321-339.

28. Anto RJ, Venkatraman M, Karunagaran D. Inhibition of NF-kappaB sensitizes A431 cells to epidermal growth factor-induced apoptosis, whereas its activation by ectopic expression of RelA confers resistance. J Biol Chem. 2003;278:25490-25498.
29. Puliyappadamba VT, Cheriyan VT, Thulasidasan AKT, et al. Nicotineinduced survival signaling in lung cancer cells is dependent on their p53 status while its down-regulation by curcumin is independent. Molecular Cancer. 2010;9:220.

30. Sreekanth CN, Bava SV, Sreekumar E, Anto RJ. Molecular evidences for the chemosensitizing efficacy of liposomal curcumin in paclitaxel chemotherapy in mouse models of cervical cancer. Oncogene. 2011;30:3139-3152.

31. Simbulan-Rosenthal CM, Rosenthal DS, Iyer S, Boulares AH, Smulson ME. Transient poly(ADP-ribosyl) ation of nuclear proteins and role of poly(ADP-ribose) polymerase in the early stages of apoptosis. J Biol Chem. 1998;273:13703.

32. Bava SV, Puliappadamba VT, Deepti A, Nair A, Karunagaran D, Anto RJ. Sensitization of taxol-induced apoptosis by curcumin involves down-regulation of nuclear factor-kappaB and the serine/threonine kinase Akt and is independent of tubulin polymerization. $J$ Biol Chem. 2005;280:6301-6308.

33. Aggarwal BB, Sung B. Pharmacological basis for the role of curcumin in chronic diseases: an age-old spice with modern targets. Trends in Pharmacol Sci. 2009;30:85-94.

34. Moses MA, Brem H, Langer R. Novel delivery systems in cancer chemotherapy. Sci Med. 2003;9:264-273.

35. Maeda H, Wu J, Sawa T, Matsumura Y, Hori K. Tumor vascular permeability and the EPR effect in macromolecular therapeutics: a review. J Control Release. 2000;65:271-284.

36. Perrault SD, Walkey C, Jennings T, Fischer HC, Chan WC. Mediating tumor targeting efficiency of nanoparticles through design. Nano Lett. 2009;9:1909-1915.

37. Mohanty C, Sahoo SK. The in vitro stability and in vivo pharmacokinetics of curcumin prepared as an aqueous nanoparticulate formulation. Biomaterials. 2010;31:6597-6611.
International Journal of Nanomedicine

\section{Publish your work in this journal}

The International Journal of Nanomedicine is an international, peerreviewed journal focusing on the application of nanotechnology in diagnostics, therapeutics, and drug delivery systems throughout the biomedical field. This journal is indexed on PubMed Central, MedLine, CAS, SciSearch ${ }^{\circledR}$, Current Contents ${ }^{\circledR} /$ Clinical Medicine,

\section{Dovepress}

Journal Citation Reports/Science Edition, EMBase, Scopus and the Elsevier Bibliographic databases. The manuscript management system is completely online and includes a very quick and fair peer-review system, which is all easy to use. Visit http://www.dovepress.com/ testimonials.php to read real quotes from published authors. 